\title{
Dificuldade na fala como preditora do declínio cognitivo na Doença de Parkinson
}

\author{
Difficulty in speech as a predictor of cognitive decline in \\ Parkinson's Disease
}

\section{Dificultad en el habla como predictor de deterioro cognitivo en la enfermedad de Parkinson}

\author{
Clarissa Evelyn Bandeira Paulino ${ }^{1}$, Hilton Justino da Silva ${ }^{2}$, Zulina \\ Souza de Lira ${ }^{3}$
}

1.Fonoaudióloga, estudante do Mestrado em Saúde da Comunicação Humana da Universidade Federal de Pernambuco (UFPE), Recife-PE, Brasil. ORCID: 0000-0002-9028-649X

2.Doutor em Nutrição e professor Associado I do curso de Fonoaudiologia da Universidade Federal de Pernambuco (UFPE), Recife-PE, Brasil. ORCID:0000-0002-6852-3233

3. Doutora em Linguística e professora Associada I do curso de Fonoaudiologia da Universidade Federal de Pernambuco (UFPE), Recife-PE, Brasil. ORCID: 0000-0002-8413-5513

\begin{abstract}
Resumo
No presente trabalho, apresentamos uma descrição crítica do artigo científico a seguir: Polychronis S, niccolini F, Pagano G, Yousaf T, Politis M. Speech difficulties in early de novo patients with Parkinson's disease. Parkinson Rel Dis 2019;64:256-61. Os autores relatam uma associação entre o déficit do neurotransmissor dopamina e a dificuldade na fala como preditora da progressão e do declínio cognitivo na Doença de Parkinson, avaliados por meio da neuroimagem e escalas de sintomas clínicos. Com base nos achados desse estudo, discutimos acerca de como essas questões podem influenciar na atenção clínica e reabilitação de sujeitos com Parkinson.
\end{abstract}

Unitermos. doença de Parkinson; fala; cognição

\begin{abstract}
In the present study, we present a critical description of the scientific article: Polychronis $\mathrm{S}$, niccolini F, Pagano G, Yousaf T, Politis M. Speech difficulties in early de novo patients with Parkinson's disease. Parkinson Rel Dis 2019;64:256-61. The authors report an association between the deficit of the neurotransmitter dopamine and speech difficulties as predictors of progression and cognitive decline in Parkinson's disease, assessed using neuroimaging and clinical symptom scales. Based on the findings of this study, we discuss how these issues can influence the clinical care and rehabilitation of subjects with Parkinson's.
\end{abstract}

Keywords. Parkinson's disease; speech; cognition

\section{Resumen}

En el presente trabajo, presentamos una descripción crítica del siguiente artículo científico: Polychronis S, niccolini F, Pagano G, Yousaf T, Politis M. Speech difficulties in early de novo patients with Parkinson's disease. Parkinson Rel Dis 2019;64:256-61. Los autores informan una asociación entre el déficit del neurotransmisor dopamina y las dificultades del habla como predictores de progresión y deterioro cognitivo en la enfermedad de Parkinson, evaluada mediante escalas de neuroimagen y síntomas clínicos. Con base en los hallazgos de este estudio, discutimos cómo estos problemas pueden influir en la atención clínica y la rehabilitación de sujetos con Parkinson.

Palabras clave. enfermedad de Parkinson; habla; cognición 


\section{RESENHA}

As alterações de fala na Doença de Parkinson (DP) compõem um quadro denominado disartria hipocinética, caracterizado por intensidade vocal reduzida, voz monótona, qualidade vocal rouca e/ou soprosa, redução na tessitura da voz falada, imprecisão articulatória, ressonância hipernasal e alterações de fluência ${ }^{1}$. Esses comprometimentos são decorrentes de sintomas motores sistêmicos característicos como tremor de repouso, rigidez muscular, instabilidade postural e lentidão na execução dos movimentos, também chamada de bradicinesia ${ }^{2}$. Repercutindo, assim, no funcionamento da respiração, fonação (voz), articulação (fala), na linguagem e na cognição ${ }^{3}$.

Com relação à cognição, recentemente a literatura vem mostrando o estudo dos aspectos demográficos e clínicos como preditores de sintomas cognitivos envolvidos na fisiopatologia da $\mathrm{DP}^{4}$. O que permite o entendimento dos sintomas oriundos do processamento neuronal e na progressão dos sintomas presentes no Parkinson já que sua causa está ligada com a diminuição de neurônios dopaminérgicos localizados nos núcleos basais ${ }^{5}$.

Diante o exposto, o estudo resenhado merece destaque $^{6}$, pois teve como hipótese a presença de dificuldades de fala como preditor de progressão motora ou 
declínio cognitivo em pessoas no estágio inicial da DP e ainda consideraram as características clínicas da doença, predominantemente rígida ou tremulante.

Nesse sentido, o estudo objetivou investigar a associação entre dificuldades de fala, déficits dopaminérgicos e progressão da doença nesses indivíduos. Para isso, foram recrutados pacientes com DP em fase inicial, com tempo de diagnóstico inferior a dois anos para um acompanhamento de três anos da progressão da doença.

O diagnóstico foi confirmado pela presença de déficit dopaminérgico utilizando Imagem FP-CIT SPECT. Para identificação de dificuldades na fala foi utilizada a escala (UPDRS-III), baseada em sintomas clínicos que consiste em 5 escores, com classificação entre 0 (normal) e 4 (comprometimento mais grave).

Após essa etapa foram identificados 143 pacientes com DP de início precoce com dificuldades de fala e pareados com 143 pacientes com DP sem dificuldades de fala que compuseram o estudo. Para a diferenciação dos grupos foram investigadas as diferenças nas características clínicas e diferenças na captação de tomografia computadorizada de emissão de fóton (SPECT) do estriado por meio do FP-CIT em pacientes com DP com e sem dificuldades de fala.

A escala UPDRS-III avaliou a gravidade dos sintomas motores utilizando os domínios bradicinesia, tremor de repouso, rigidez, instabilidade postural e seus subitens como a constância do tremor, região da rigidez (pescoço, membros superiores e inferiores), a amplitude do tremor em repouso 
nos lábios, mandíbula e membros, entre outros aspectos. Tais pontuações, na referida escala, permitiram identificar os fenótipos motores da DP classificados em tremor-dominante, acinético-rígido ou misto. Por meio de outros protocolos, foram avaliados ainda, os sintomas não motores, a função autônoma, os sintomas neuropsiquiátricos, os distúrbios do sono, a incapacidade e disfunções do olfato.

O comprometimento cognitivo foi medido usando a avaliação cognitiva de Montreal (MoCA). Por fim, as imagens dopaminérgicas pelo SPECT foram interpretadas $e$ quantificadas. Para isso, os volumes de imagem SPECT foram normalizados, oito fatias axiais mais proeminentes contendo o estriado foram somadas e em seguida, um modelo padronizado de volume de interesse (VOI) foi aplicado a esta imagem. As análises de VOI foram realizadas no caudado esquerdo e direito e putâmen com a região occiptal servindo como tecido de referência.

Como mencionado, os pacientes em fase inicial da doença foram acompanhados por um período de três anos, por meio de visitas anuais nas quais eram feitas as avaliações da progressão motora e declínio cognitivo. A progressão motora foi definida como uma mudança de ponto na escala $H \& Y$, medida no estado "off" do tratamento. $O$ comprometimento cognitivo foi definido como escore $\leq 22$ no MoCA. Na análise dos dados, foi adotado o valor de $p<0,05$, para todas as comparações.

Como resultados do estudo destacam-se que, dos 143 pacientes com dificuldades na fala, 137 apresentaram 
dificuldade leve e 6 dificuldade moderada e estes no exame de imagem, no estágio inicial, exibiram menor captação de FP-CIT no estriado, caudado e putâmen em comparação ao grupo sem dificuldades de fala, tais achados, ainda se relacionaram a piores escores de fala mensurados pelo UPDRS-III. Com relação à progressão da doença, 42,8\% dos pacientes em estágio inicial apresentaram evolução dos sintomas motores e 7,6\% evoluíram com prejuízo cognitivo.

Quando incluídas as covariáveis idade e sexo em uma análise de risco proporcional, observou-se que a presença de dificuldades de fala em pacientes com DP em fase inicial previa o declínio cognitivo ao longo de três anos, contudo não influenciava na progressão motora. Esses pacientes logo apresentaram também, maior disfunção autonômica e aumento da sonolência diurna. Ressalta-se a importância de chamar a atenção para estes sintomas como manifestações sutis que podem ser identificados na DP em fase inicial, mas que não são considerados como queixa, dada a relevância dos sintomas motores considerados para o diagnóstico?.

Nesse contexto, os achados do estudo permitem inferências clínicas, pois levam em consideração aspectos cognitivos da doença como agentes envolvidos na dificuldade de fala. O que pode ocorrer devido à lentidão do processamento das informações centrais e não somente das alterações da motricidade orofacial oriundas da atividade muscular como discutido no próprio artigo. Originárias, portanto, de danos no planejamento motor e dificuldades na execução de programas simultâneos ou sequenciais próprios 
de disfunções dos núcleos da base, já que, o controle motor da fala se encontra comprometido em razão da disartria hipocinética caracterizando dentre outros aspectos, prejuízos na expressão verbal, articulação imprecisa, disprosódia, disfluência, qualidade vocal alterada, monotonia na fala 8 .

As dificuldades de fala mais comuns foram encontradas no tipo acinético-rígido, o que pode sugerir relação direta desse fator com a bradicinesia e rigidez dos músculos laríngeos. A estrutura laríngea é impactada pela musculatura rígida decorrente das alterações neuromusculares próprias da DP, resultando na instabilidade vibratória das pregas vocais e consequente prejuízo da fala ${ }^{9}$. Esse dado corrobora a atenção clínica frente à manipulação muscular e, sobretudo, na seleção de técnicas vocais e de articulação pertinentes a essa condição, levando em conta que as dificuldades de fala são uma característica debilitante comum na DP. Nesse contexto, foi citado pelos autores estudos que mostraram a ativação do circuito dos núcleos da base, cerebelo e córtex associados ao recrutamento da região motora orofacial em indivíduos com Parkinson.

Concluíram ainda que as dificuldades de fala estão associadas a déficits dopaminérgicos do estriado, possibilitando também a inferência dos substratos neurais envolvidos na produção da fala em sujeitos com DP e permitindo o entendimento das causas de redução da entonação, prosódia, ritmo, imprecisão articulatória e da fluência verbal como outros estudos também sugerem ${ }^{10}$. 
Em contrapartida, algumas reflexões acerca desse artigo podem ser feitas com base nas limitações pontuadas pelos autores, por exemplo, a ausência de avaliações vocais acústicas para avaliar dificuldades de fala em pacientes com DP. Inclusive, os próprios autores discutem outros trabalhos que utilizaram avaliação vocal acústica quantitativa mostraram que variação da frequência fundamental da voz e o índice de ritmicidade de fala podem prever mudanças no estado cognitivo ${ }^{11}$.

Sugeriram, ainda, a possibilidade de novos estudos utilizando esses parâmetros acústicos da voz e a avaliação perceptivoauditiva por fonoaudiólogo para melhor entendimento dessas questões, já que o instrumento utilizado no estudo identificou dificuldades de grau leve em sua maioria. A análise vocal por meio do parâmetro perceptivo auditivo é um tipo de avaliação que permite que um fonoaudiólogo treinado identifique características nas vozes, que podem ser sugestivas de patologias orgânicas, funcionais ou neurológicas ${ }^{12}$.

Finalmente, os pontos aqui destacados nos levam a refletir sobre a terapia com pacientes com a DP, sobretudo, fonoaudiológica, na qual se tem a base da estimulação pautada em comandos a serem desempenhados/reproduzidos pelos pacientes. Conhecer os padrões de reprodução muscular para a comunicação oral por meio da fisiopatologia da doença e saber que a fonte da execução de um exercício ou da produção da fala, propriamente dita, é oriunda das funções cognitivas e do 
processamento central e depende da praxia motora permite a condução de direcionamentos terapêuticos. A técnica de sobrearticulação de fala, por exemplo, é um grupo de exercícios que demanda um recrutamento das fibras musculares a partir do entendimento da técnica pelo paciente para realizar o comando neural, se bem desempenhado, produz efeito positivo imediato nos aspectos vocais e uma maior expressividade facial em sujeitos com $\mathrm{DP}^{13}$.

Nesse sentido, dada a importância de trabalhar a fala em sujeitos com DP, sobretudo na atuação fonoaudiológica, entende-se que essa função pode contribuir com além da melhora da comunicação oral, a cognição desses indivíduos. Podendo, os exercícios para voz, articulação e prosódia, estimular os processos cognitivos e por conseguinte, minimizar a progressão da Doença de Parkinson e promover melhora da qualidade de vida.

\section{Agradecimentos}

Coordenação de Aperfeiçoamento de Pessoal de Nível Superior (CAPES), código de financiamento 001.

\section{REFERÊNCIAS}

1.Brabo NC, Minett TSC, Ortiz KZ. Fluency in Parkinson's disease: disease duration, cognitive status and age. Arq Neuropsiquiatr 2014;72:349-55. 
https://doi.org/10.1590/0004-282X20140018

2.Cruz AND, Beber BC, Olchik MR, Chaves MLF, Rieder CRDM, Dornelles

$\mathrm{S}$. Aspectos de comunicação oral em pacientes com doença de

Parkinson submetidos à Estimulação Cerebral Profunda. CoDAS São

Paulo 2016;28:480-5. https://doi.org/10.1590/2317-

$1782 / 20162015169$

3. Ortiz KZ. Disartria. In: Ortiz KZ. Distúrbios neurológicos adquiridos: fala e deglutição. Barueri: Manole; 2006, p.54-71.

4. Martins NM, Asano NMJ, Lins CCDSA, Coriolano MDGWD. Variáveis demográficas e clínicas como preditoras diferenciais de alteração cognitiva na doença de Parkinson. Rev Bras Geriatr Gerontol 2019;22:e180141.

https://doi.org/10.1590/1981-22562019022.180141

5.Cunha C, Wietzikoski EC, Dombrowski P, Bortolanza M, Santos $\mathrm{LM}$, Boschenet $\mathrm{SL}$, et al. Learning processing in the basal ganglia: a mosaic of broken mirors. Behav Brain Res 2009;199:157-70. https://doi.org/10.1016/j.bbr.2008.10.001

6. Polychronis S, niccolini F, Pagano G, Yousaf T, Politis M. Speech difficulties in early de novo patients with Parkinson's disease. Parkinson Rel Dis 2019;64:256-61.

https://doi.org/10.1016/j.parkreldis.2019.04.026

7. Ferraz HB, Silva CC. Unusual early symptoms of Parkinson's disease. Why do we need to identify them? Arq Neuropsiquiatr 2016;74:77980. https://doi.org/10.1590/0004-282X20160135

8.Presotto $M$, Rieder CRM, Olchik MR. Validação de conteúdo e confiabilidade do Protocolo de Avaliação dos Distúrbios Adquiridos de Fala em Indivíduos com Doença de Parkinson (PADAF). CoDAS 2019; 31:e20180230. https://doi.org/10.1590/2317-1782/20192018230

9.Logemann JA, Fisher HB, Boshes B, Blonsky ER. Frequency and cooccurrence of vocal tract dysfunctions in the speech of a large sample of Parkinson patients. J Speech Hear Disord 1978;43:47-57.

https://doi.org/10.1044/jshd.4301.47

10.El-Nazer R, Adler CH, Beach TG, Belden CM, Artz J, Shill HA, et al. Regional neuropathology distribution and verbal fluency impairments in Parkinson's disease. Parkinson Rel Dis 2019;65:73-8.

https://doi.org/10.1016/j.parkreldis.2019.05.014

11.Rektorova I, Mekyska J, Janousova E, Kostalova M, Eliasova I, Mrackova $M$, et al. Speech prosody impairment predicts cognitive decline in Parkinson's disease. Parkins Rel Dis 2016;29:90-5. https://doi.org/10.1016/j.parkreldis.2016.05.018

12.Behlau M, Madazio G, Feijó D, Pontes P. Avaliação de voz. In: Behlau M. Voz: o livro do especialista. Rio de Janeiro: Revinter; 2001. p.85-246.

13.Bento FAM, Diaféria GLA, Fonoff ET, Padovani MMP, Behlau M. Efeito da técnica de sobrearticulação na voz e na fala em indivíduos com doença de Parkinson após cirurgia de estimulação cerebral profunda. Audiol Commun Res 2019;24:e2008.

https://doi.org/10.1590/2317-6431-2018-2008 